\section{Identification and quantitation of blasts in myeloid malignancies with marrow fibrosis or marrow hypoplasia and CD34 negativity}

Sir,

Despite the advent of molecular genetics, a commonly asked question is 'what is the percentage of blasts in the marrow?' Blast percentage is one of the criteria for diagnosis and classification of myelodysplastic syndromes and acute leukaemias, and for subtyping of acute myeloid leukaemias (e.g., myelomonocytic leukaemia, pure erythroid leukaemia) and myeloproliferative/myelodysplastic neoplasms (e.g., chronic myelomonocytic leukaemia). It also forms part of the definition of morphological remission in acute leukaemias as well as the definition of accelerated or blast phases of myeloproliferative neoplasms. Blast percentage bears prognostic significance in various haematological neoplasms as it is part of various scoring systems [e.g., International Prognostic Scoring System (IPSS)]. Diagnosis of relapse of the abovementioned neoplasms also depends on accurate blast quantitation. Lastly, it is not uncommon to see circulating 'immature' cells and having to differentiate between true blasts from circulating lymphoma or other immature cells can be challenging.

Estimation of an accurate blast count is not always simple or straightforward, particularly in the following settings:

1. An aparticulate bone marrow aspirate or 'dry tap' makes the estimation of blast count challenging because:

- The aspirate blast count may not be representative as a result of poor technical quality of the aspirated specimen or as a result of marrow fibrosis.

- The blast cell morphology on the trephine imprint is not always optimal or conducive to obtaining an accurate blast count.

- Blast quantitation is difficult to estimate on a trephine biopsy, especially when the involvement is patchy, not extensive, or in the setting of a hypocellular marrow. In such cases immunostaining with 'progenitor' cell markers (e.g., CD34, CD123 and CD117 in conjunction with E-cadherin) may be helpful.

2. The bone marrow in a patient with acute leukaemia recovering following induction chemotherapy often has an excess of immature cells, including left-shifted myelopoiesis and early erythroblasts. In such cases distinguishing early erythroblasts from residual leukaemic blasts is required but often challenging.

3. Blasts can be difficult to distinguish from dysplastic promyelocytes and myelocytes. This is not an uncommon problem particularly in patients with chronic myelomonocytic leukaemia (CMML), multiply-treated relapsed acute myeloid leukaemia (AML), therapy-associated myeloid neoplasms, or AML with myelodysplastic related changes. Additionally, new targeted therapies that induce differentiation such as IDH inhibitors also add to these morphological difficulties.

4. In CMML and acute leukaemias of monocytic lineage, distinction between blasts, promonocytes and monocytes can be subjective and notoriously difficult.
5. A $5 \%$ blast count could vary from $1-12 \%$ if only 100 WBCs cells are counted, compared to $4.6-5.4 \%$ if 10,000 cells are counted.

6. Technical issues with the quality of staining may compromise morphological blast estimation on Romanowsky stained aspirate smears and H\&E stained trephine biopsies.

Although the solution needs to be individualised, one or more of the following are often helpful:

1. Ensuring excellent technical staining quality of aspirate smears and H\&E trephine biopsy sections.

2. Seeking an opinion from someone more experienced.

3. Establishing a blast count based on at least a 500-cell differential given the associated greater accuracy and a tighter confidence interval. ${ }^{1}$ A differential count based on at least 500 cells on a bone marrow aspirate stained with an appropriate Romanowsky stain is recommended as standard practice for the diagnosis and classification of myeloid neoplasms. ${ }^{2}$

4. Detecting a blast count of $>20 \%$ on peripheral blood in a patient with a 'dry bone marrow aspirate tap' and thus fulfilling the criteria for a diagnosis of acute leukaemia.

5. Determining when quantitation of blasts may not be required for prognostication. For example, it was shown in one study that in therapy-related myeloid neoplasms, blast count is not a predictor of outcomes once it is $>5 \%$. Therefore, the need to quantify blasts accurately in this setting may not be critical.

6. Correlation with flow cytometry when there is uncertainty regarding the nature of blasts to confirm their identity and distinguish them from other cells (e.g., blastic mantle lymphoma cells). However, it is important to note that blast quantitation on bone marrow aspirates by flow cytometry is routinely underestimated when compared to morphological assessment because flow cytometry samples are invariably diluted with blood.

7. Determining situations where the $5 \%$ blast count cut-off may not be critical (e.g., defining complete remission following induction therapy in AML). Patients with $\geq 5 \%$ blasts on morphology in the context of regenerating bone marrow samples may have no leukaemic blasts identified on flow cytometry; conversely patients with $<5 \%$ blasts on morphology may still have detectable minimal residual disease on flow cytometry. ${ }^{4,5}$ In fact some recent studies question the validity of defining morphological remission from AML ( $<5 \%$ blasts). Instead they recommend that complete remission in AML could be better defined by 'first pull' aspirate analysed by flow cytometry which demonstrates the absence of phenotypically abnormal blasts, rather than by the percentage of blasts. ${ }^{6}$

Immunostaining on trephine biopsy for CD34 is the most consistent and practical method of identifying and quantifying blasts using visual estimation. In our laboratory we quantify blasts within a range and as a percentage of total marrow cellularity; for example, $\sim 5 \%, 10-20 \%$ or $\sim 20 \%$, depending upon the estimated percentage positivity, as we have previously validated the robustness of such quantitation on plasma cell myeloma immunohistochemistry. However, in our experience it is not infrequent to see cases where the 
blast percentage is low in the bone marrow aspirate but immunostaining of the trephine biopsy demonstrates focal collections of CD34 and/or CD117 or CD123 positive blasts. Furthermore, CD34 expression can be absent (defined as $<20 \%$ of blasts expressing CD34) in up to $50 \%$ of newly diagnosed cases of AML. ${ }^{8}$ We have been exploring the role of extended immunohistochemistry in cases where blast identification and quantitation have proven to be difficult on morphology and/or flow cytometry for one reason or another. In such cases, and in those where the blasts are CD34 negative, immunostaining for CD123 and CD117 can be useful.

CD117 is an immature myeloid cell marker that stains myeloblasts, promyelocytes and early erythroblasts. In our experience, blasts are often weakly stained with CD117 in trephine sections when compared to brightly staining normal mast cells. Distinguishing early erythroblasts from myeloblasts can be facilitated by the concurrent examination of an E-cadherin stain for early erythroblast estimation given that this protein is specifically expressed in early erythroblasts with gradual downregulation as erythroid cells mature. However it can be almost impossible to differentiate promyelocytes from myeloblasts when evaluating a CD117 immunostained slide, thus making blast estimation challenging in certain myeloid neoplasms.

CD123 is another marker of early haematopoietic progenitor cells that can be useful in detecting blasts. It has been shown to be positive in up to $90 \%$ of AML cases depending upon the cut-off for definition of positivity (5$20 \%) .{ }^{10,11}$ AML has the highest percentage of CD123 positive cells amongst the acute leukaemias with at least
$80 \%$ of leukaemic cells expressing the marker, making it a robust tool for blast cell identification and estimation. ${ }^{11}$ Like most immunohistochemical markers one needs to be aware of the positivity of non-blast cells, e.g., plasmacytic dendritic cells, basophils, eosinophils, megakaryocytes, and some B cells. The distinction between blasts and nonblast cells can be mostly made on morphology of the immunostained sections by cytological features. An example where this might be useful is the distinction of blasts from basophils in CML to determine the phase of disease. In our experience, CD123 has been particularly useful in confirming blast quantitation in CD34 negative cases of AML. A recent study has also shown CD123 to be a worse prognostic marker for normal karyotypic AML, likely due to its correlation with FLT3-ITD. ${ }^{12}$ In addition, it is currently being investigated as a potential immunotherapeutic target.

In our experience there are a small number of AML cases in which the blasts are negative for all three progenitor markers, i.e., CD34, CD123 and CD117; in such cases, identification and quantitation of blasts can be especially difficult. However, the relative utility of these antibodies is illustrated in Fig. 1-3.

In summary, for cases in which blast enumeration is not representative on bone marrow aspirate smears, particularly due to marrow fibrosis or hypocellularity, immunostaining with CD34 can be helpful in quantifying blasts. When the blasts are CD34 negative, immunostaining of trephine sections for CD123 and CD117 may be helpful in estimating a semi-quantitative blast percentage and should aid in the
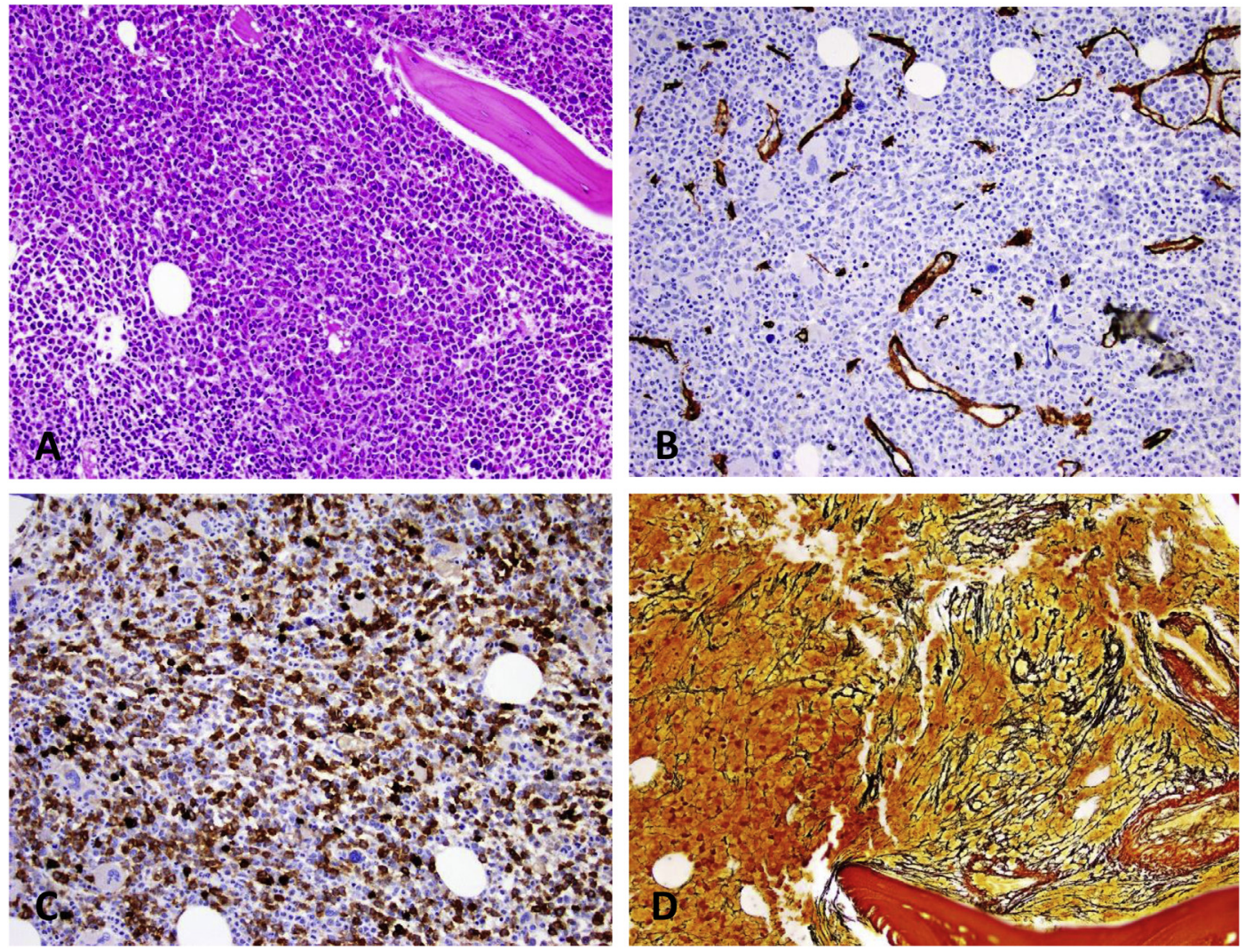

Fig. 1 Newly diagnosed AML with fibrosis. The aspirate was aparticulate as a result of the myelofibrosis (MF 2-3) but the trephine biopsy demonstrated an excess of blasts, which were CD34 negative but CD123 positive. (A) H\&E; (B) CD34; (C) CD123; (D) reticulin stain. 


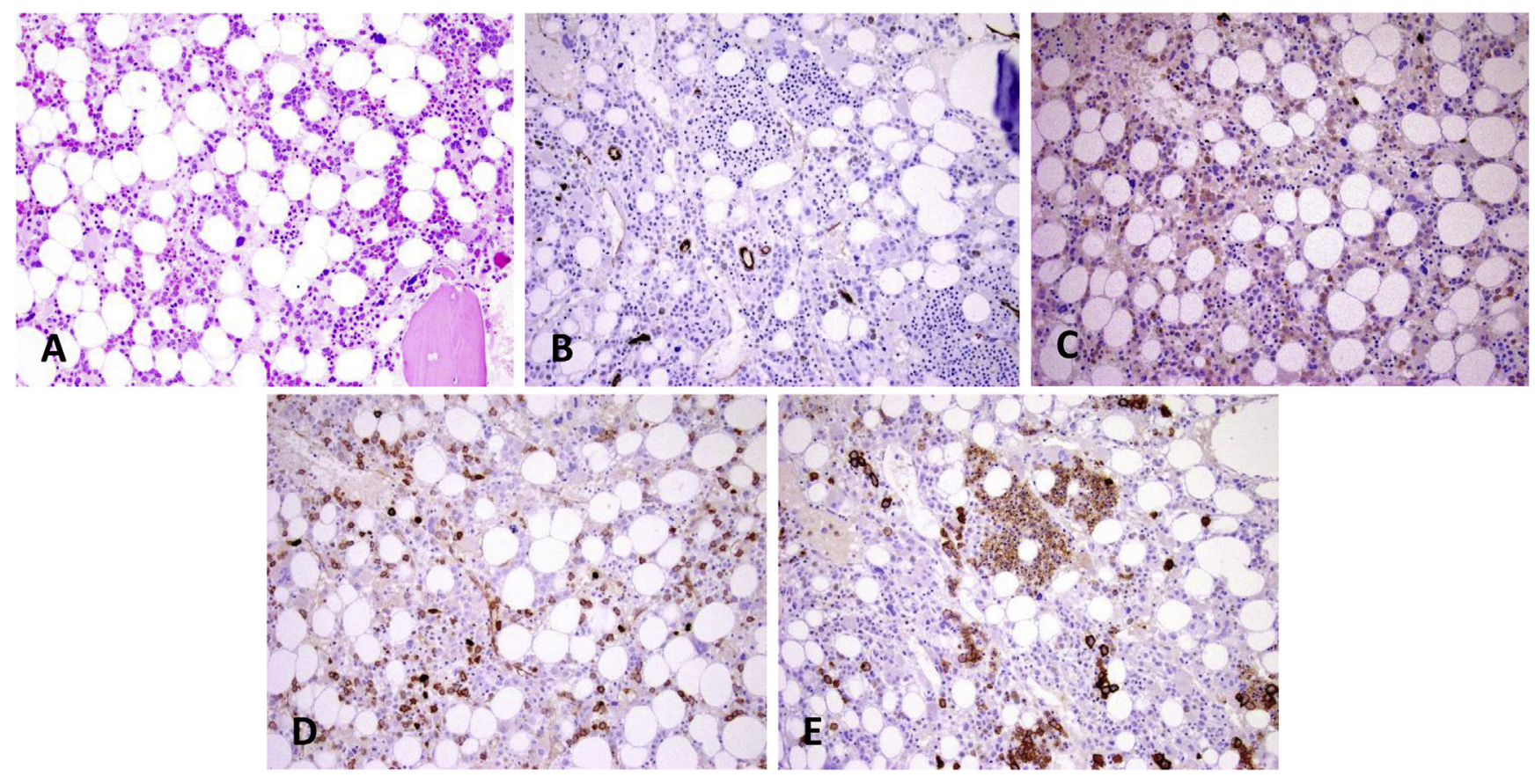

Fig. 2 (A) Relapsed AML with hypocellular marrow (H\&E) and 'difficult to quantify' blasts which are (B) CD34 negative, (C) CD117 dimly positive, but (D) CD123 positive. (E) Scattered individual cells as well as clusters of E-cadherin positive erythroblasts were seen. Although the aspirate revealed $17 \%$ blasts, a diagnosis of relapsed AML was made based on CD123 immunostaining $(>20 \%)$.
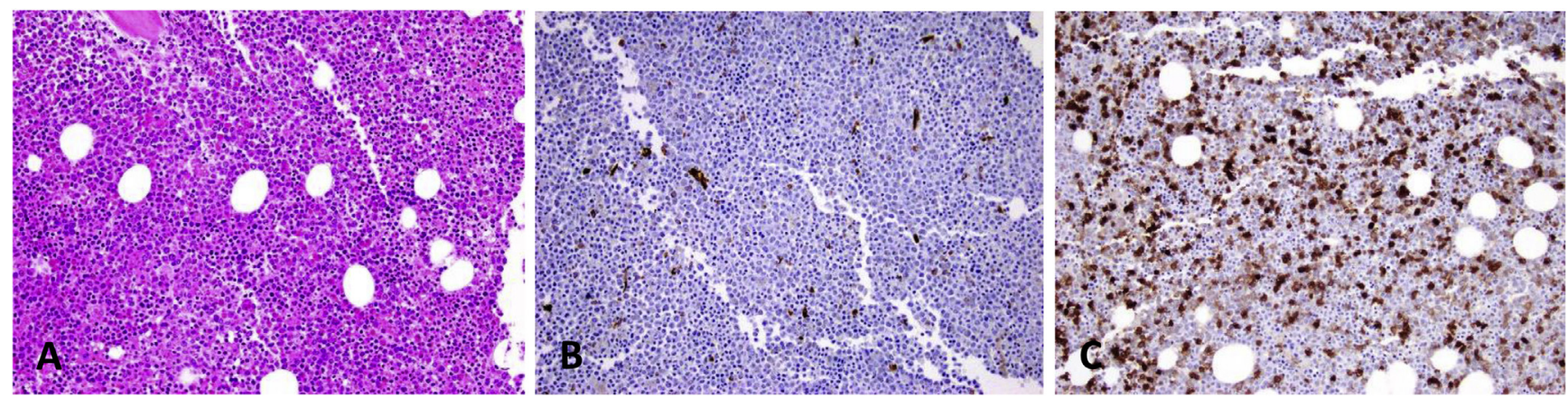

Fig. 3 Refractory AML following IDH1-inhibitor therapy. Assessment of the aspirate Romanowsky enumerated 14\% blasts and (A) the H\&E stain demonstrated an interstitial excess of blasts that was difficult to quantify. (B) CD34 staining only demonstrated weak positivity ( $\sim 5 \%)$ but (C) CD123 staining showed $>20 \%$ positivity, confirming refractory disease.

correct diagnosis and classification of various haematological neoplasms.

Conflicts of interest and sources of funding: The authors state that there are no conflicts of interest to disclose.

\section{Rithin Nedumannil $^{1}$, Shirlene Sim $^{1}$, David Westerman ${ }^{1,2}$, Surender Juneja ${ }^{1,2,3}$}

${ }^{1}$ Department of Haematopathology, Peter MacCallum Cancer Centre, Melbourne, Vic, Australia; ${ }^{2}$ University of Melbourne, Parkville, Vic, Australia; ${ }^{3}$ Department of Diagnostic Haematology, Royal Melbourne Hospital, Parkville, Vic, Australia

Contact Dr Rithin Nedumannil.

E-mail: rithin.nedumannil@ petermac.org

1. Briggs C, Bain B. Differential count. In: Bain BJ, Bates I, Laffan MA, editors. Dacie and Lewis Practical Haematology. 5th ed. London: Elsevier, 2017.
2. Swerdlow SH, Campo E, Harris NL, editors. WHO Classification of Tumours of Haematopoietic and Lymphoid Tissues. 4th ed. Lyon: IARC, 2017.

3. Ok CY, Hasserjian RP, Fox PS, et al. Application of the international prognostic scoring system-revised in therapy-related myelodysplastic syndromes and oligoblastic acute myeloid leukemia. Leukemia 2014; 28: 185-9.

4. Chen X, Xie H, Wood BL, et al. Relation of clinical response and minimal residual disease and their prognostic impact on outcome in acute myeloid leukemia. J Clin Oncol 2015; 33: 1258-64.

5. Ouyang J, Goswami M, Tang G, et al. The clinical significance of negative flow cytometry immunophenotypic results in a morphologically scored positive bone marrow in patients following treatment for acute myeloid leukemia. Am J Hematol 2015; 90: 504-10.

6. Araki D, Wood BL, Othus M, et al. Allogeneic hematopoietic cell transplantation for acute myeloid leukemia: time to move toward a minimal residual disease-based definition of complete remission? J Clin Oncol 2016; 34: 329-36.

7. Ng A, Wei A, Bhurani D, Chapple P, Feleppa F, Juneja S. CD138 immunostaining of bone marrow trephine specimens is the most sensitive method for quantifying marrow involvement in patients with low percentage of plasma cells. Haematologica 2006; 91: 972-5.

8. Raspadori D, Lauria F, Ventura MA, et al. Incidence and prognostic relevance of CD34 expression in acute myeloblastic leukemia: analysis of 141 cases. Leuk Res 1997; 21: 603-7.

9. Armeanu S, Müller CA, Klein G. Involvement of E-cadherin in the development of erythroid cells. Hematology 2000; 5: 307-16. 
10. Das N, Gupta R, Gupta S, et al. A real-world perspective of CD123 expression in acute leukaemia as promising biomarker to predict treatment outcome in B-ALL and AML. Clin Lymphoma Myeloma Leuk 2020; 20: e673-84.

11. Bras AE, Haas V, Stigt A, et al. CD123 expression levels in 846 acute leukemia patients based on standardised immunophenotyping. Cytometry B Clin Cytom 2019; 96B: 134-42.

12. Jiang G, Atenafu EG, Capo-Chichi JM, et al. Prognostic relevance of CD123 expression in adult AML with normal karyotype. Br J Haemato 2020; 188: $181-4$

DOI: https://doi.org/10.1016/j.pathol.2020.10.022

\section{Abnormal coagulation profiles in monoclonal gammopathy of undetermined significance: a case series}

Sir,

Monoclonal gammopathy of undetermined significance (MGUS) is a plasma cell disorder characterised by a serum monoclonal protein of less than $30 \mathrm{~g} / \mathrm{L}$, clonal bone marrow plasma cells (or lymphoplasmacytic infiltration) of less than $10 \%$ and no evidence of end organ damage. ${ }^{1}$ Plasma cell dyscrasias can be complicated by disordered haemostasis; in particular an increased risk of thrombosis is seen. ${ }^{2}$ However, a bleeding diathesis has also been reported in some patients, and abnormal coagulation study results are seen relatively commonly in patients with multiple myeloma. ${ }^{3-5}$ We report three patients referred for investigation of a prolonged activated partial thromboplastin time (APTT) who were identified to have an underlying MGUS.

The first patient was a 69 -year-old male who was referred for assessment with an incidentally detected APTT of 45 [normal range (NR) 25-35 s] and PT of 13 (NR 11-15 s) at anaesthetic pre-admission clinic. This patient had been diagnosed with a caecal tumour and had a planned robotic right hemicolectomy. He had previous surgical procedures including basal cell carcinoma removal, metatarsal shave procedure and tonsillectomy and adenoidectomy as a child, none of which were complicated by bleeding. He reported some spontaneous protracted nosebleeds approximately 20 years earlier, but no other significant bleeding. His International Society of Thrombosis and Haemostasis-Bleeding Assessment Tool (ISTH-BAT) was 1 (normal range <4). He was not receiving any antiplatelet or anticoagulant therapy.

Further investigation demonstrated that his APTT did not correct to the normal range on mixing (36 s). Specific factor level testing demonstrated a Factor XI concentration of $15 \%$ at 1:10 dilution (Table 1). With serial dilutions of plasma, all factor levels increased, suggesting possible inhibition of all factors assessed; however, this was most marked with Factor XI, which increased to a level of $60 \%$ with a 1:200 dilution (Table 1). A specific Factor XI inhibitor could not be demonstrated on Bethesda assay including a $2 \mathrm{~h}$ incubation. A lupus inhibitor was not detected. Platelet function assay100 (PFA-100) testing on this patient was normal.

Serum electrophoresis and immunofixation were performed and demonstrated an IgG kappa paraprotein measuring $3 \mathrm{~g} / \mathrm{L}$. Serum free light chain assessment demonstrated a kappa free light chain of $182 \mathrm{~g} / \mathrm{L}$ and lambda of
$14.92 \mathrm{~g} / \mathrm{L}$ (ratio 14.92). Flow cytometry of the peripheral blood demonstrated an abnormal B-cell population with a clone size of $0.3 \times 10^{9} / \mathrm{L}$ that was positive for CD19, CD20 (moderate-bright intensity), kappa (dim-moderate intensity), CD22 (dim), CD200, and negative for CD5, CD10, CD11c, CD23. There was no renal impairment, hypercalcaemia, anaemia or bony disease on a skeletal survey. Bone marrow biopsy had not been performed as we were awaiting completion of his adjuvant chemotherapy.

The factor assay findings were suspected to be secondary to interference by the $\mathrm{M}$ protein. He proceeded to his robotic right hemicolectomy with four units of fresh frozen plasma (FFP) thawed in case of bleeding. The operation proceeded without complication and FFP administration was not required. His APTT remained prolonged after resection of the caecal tumour and six cycles of adjuvant chemotherapy. His Factor XI remained low (24\% at 1:10 dilution), and all factor levels increased with serial dilution of plasma (Table 1). von Willebrand factor (VWF) studies demonstrated a VWF antigen of $172 \mathrm{U} / \mathrm{dL}$ (NR 50-200), VWF ristocetin cofactor of $188 \mathrm{U} / \mathrm{dL}$ (NR 45-200) and VWF collagen of $>200 \mathrm{U} / \mathrm{dL}$ (NR 50-200). Of note, on follow-up this patient now has two detectable IgM kappa paraproteins in addition to his IgG kappa paraprotein.

The second patient was a 70-year-old female who was referred for assessment of a prolonged APTT of $51 \mathrm{~s}$ which did not correct on mixing ( $49 \mathrm{~s}$ ) on a background of a known MGUS. She presented for elective insertion of a permanent pacemaker. She had no spontaneous bruising or bleeding but reported previous prolonged, delayed and moderately severe bleeding post-surgical procedures including prolonged bleeding following tooth extractions requiring hospital admission, post-partum haemorrhage and post-operative bleeding following dilatation and curettage, laparoscopic cholecystectomy and hysterectomy. She was not receiving any antiplatelet or anticoagulant therapy.

Intrinsic Factor assessment at 1/10 dilution demonstrated a Factor VIII of $3 \%$, Factor IX of $1 \%$, Factor XI of $1 \%$ and Factor XII of $4 \%$ using one stage factor assays (Table 1 ). These demonstrated some improvement with plasma dilution. A two stage chromogenic Factor VIII assay demonstrated a much higher level of $114 \%$ (Table 1). A specific factor inhibitor could not be identified on Bethesda assay. VWF studies were normal as were platelet count and platelet aggregometry. A lupus inhibitor screen was negative. Paraprotein assessment demonstrated an IgA lambda paraprotein measuring $7 \mathrm{~g} / \mathrm{L}$.

Given her history of bleeding, this patient received four units of fresh frozen plasma prior to the permanent pacemaker insertion but had no intra-operative or post-operative bleeding. She was then lost to follow-up for several years but referred back for assessment after having delayed bleeding following tooth extractions. Repeat coagulation studies were unchanged compared with her studies 7 years prior. In addition, her paraprotein was stable.

The third patient was a 46-year-old male who presented with minor bleeding not requiring intervention related to low risk surgical procedures (minor liposuction, removal of axillary sweat glands) and was noted to have a prolonged APTT of $57 \mathrm{~s}$ that did not correct on mixing (55 s). He was not receiving any antiplatelet or anticoagulant therapy. Factor level testing demonstrated a reduction in levels of all the 